\title{
Feasibility of Noninvasive Continuous Finger Arterial Blood Pressure Measurements in Very Young Children, Aged 0-4 Years
}

\author{
PETER ANDRIESSEN, OLAF SCHRAA, WENDY VAN DEN BOSCH-RUIS, DERK JAN TEN HARKEL, JOS J. SETTELS, \\ SIDARTO BAMBANG OETOMO, AND CARLOS E. BLANCO
}

\begin{abstract}
Neonatal Intensive Care Unit [P.A., S.B.O.], Máxima Medical Center, 5500 MB Veldhoven, The Netherlands; Cardiovascular Monitoring [O.S., J.J.S.], BMEYE, Academic Medical Center, 1105 AZ Amsterdam, The Netherlands; Department of Pediatrics [W.B.-R.], Spaarneziekenhuis, 2134 TM Hoofddorp, The Netherlands; Department of Pediatric Cardiology [D.J.H.], Erasmus Medical Center, 3015 GJ Rotterdam, The Netherlands; Department of Industrial Design [S.B.O.], Eindhoven University of Technology, 5612 AZ Eindhoven, The Netherlands; Department of Pediatrics [C.E.B.], Academic Hospital Maastricht, 6229 HX Maastricht, The Netherlands
\end{abstract}

\begin{abstract}
Our goal was to study the feasibility of continuous noninvasive finger blood pressure (BP) monitoring in very young children, aged 0-4 y. To achieve this, we designed a set of smallsized finger cuffs based on the assessment of finger circumference. Finger arterial BP measured by a volume clamp device (Finapres technology) was compared with simultaneously measured intra-arterial $\mathrm{BP}$ in 15 very young children (median age, 5 mo; range, 0-48), admitted to the intensive care unit for vital monitoring. The finger cuff-derived BP waveforms showed good resemblance with the invasive arterial waveforms (mean root-mean-square error, $3 \mathrm{~mm}$ $\mathrm{Hg}$ ). The correlation coefficient between both methods was $0.79 \pm$ 0.19 systolic and $0.74 \pm 0.24$ diastolic. The correlation coefficient of beat-to-beat changes between both methods was $0.82 \pm 0.18$ and $0.75 \pm 0.21$, respectively. Three measurements were related to measurement errors (loose cuff application; wrong set-point). Excluding these erroneous measurements resulted in clinically acceptable measurement bias $(-3.8 \mathrm{~mm} \mathrm{Hg})$ and $95 \%$ limits of agreement $(-10.4$ to $+2.8 \mathrm{~mm} \mathrm{Hg})$ of mean $\mathrm{BP}$ values. We conclude that continuous finger BP measurement is feasible in very young children. However, cuff application is critical, and the current set-point algorithm needs to be revised in very young children. (Pediatr Res 63: 691-696, 2008)
\end{abstract}

$I^{\mathrm{n}}$ n human adults, continuous noninvasive finger blood pressure (BP) measurement has been available using the Finapres (FINger Arterial PRESsure) device (1). It has been widely used in the investigation of autonomic function in adults and has proven to be a reliable substitute for intraarterial measurements (2). The device is based on a photoplethysmographic system applied to the finger and provides beat-to-beat arterial finger BP with the use of the volume clamp technique of Peñáz (3). The Finapres method is evaluated in children aged 6-16 y, showing similar accuracy as that in adults (4,5). Because no appropriate finger cuff exists for the children below $6 \mathrm{y}$ of age, the applicability of the method

Received August 29, 2007; accepted January 8, 2008

Correspondence: Peter Andriessen, M.D., Ph.D., Máxima Medical Center, Neonatal Intensive Care Unit, PO Box 7777, 5500 MB Veldhoven, The Netherlands; e-mail: p.andriessen@mmc.nl

PA, WB, AJDH, SBO and CEB have no financial interest, (sponsor) arrangement, or affiliation with BMEYE, other than participation in the present study. is limited in this age group. Still, in the evaluation of pediatric patients with cardiovascular abnormalities (e.g. sepsis) or autonomic dysfunction (e.g. syncope), it is desirable to have continuous BP measurements.

Recently, there are several publications in which investigators performed BP measurements with the Finapres method in newborns by simply applying the (commercially available) finger cuff around the wrist (6-9). However, Finapres was not designed for BP measurements on the wrist. First, the method of volume clamping is only validated with an appropriate cuff applied to the midphalanx of the finger. Second, the difference between fingers of adults and children may not only be the size but also the physiologic properties of the arteries and thus need an adaptation of the autocalibration (algorithm). Third, applying the cuff around the wrist will result in venous congestion of the whole hand and not just the fingertip, and will ultimately limit its clinical applicability. Measurements on adult fingers have been performed for prolonged periods ( 24 h) with no reports of lasting damage (10).

Therefore, the aim of the study was to investigate the feasibility of noninvasive finger BP measurement in newborns, infants, and young preschool children. To achieve this we developed different prototypes of miniature finger cuffs. Our primary goal was to determine whether it is possible to obtain representative arterial BP waveforms in this age group with very small fingers. In addition, we wanted to study whether the finger BP measurements were well tolerated by the children. Finally, noninvasive finger BP measurements were compared with intra-arterial $\mathrm{BP}$ values to estimate the accuracy of the noninvasive technique.

\section{METHODS}

Subjects. The study was performed in five full-term newborns, four infants (1-12 mo), and six preschool children (1-4 y) admitted to a neonatal (Máxima Medical Center, Veldhoven, The Netherlands) or pediatric intensive care unit (Emma Children's Hospital, Academic Medical Center, Amsterdam or Sophia Children's Hospital, Erasmus Medical Center, Rotterdam, The Netherlands) whose intensive care management required an indwelling arterial catheter. The clinical characteristics of the population are shown in Table 1.

Abbreviations: BP, blood pressure; RMSE, root-mean-square error 
Table 1. Characteristics of the study population

\begin{tabular}{cccclc}
\hline $\begin{array}{c}\text { Subject } \\
\text { no. }\end{array}$ & $\begin{array}{c}\text { Weight } \\
(\mathrm{kg})\end{array}$ & Age & $\begin{array}{c}\text { Cuff } \\
\text { size }\end{array}$ & $\begin{array}{c}\text { Primary } \\
\text { diagnosis }\end{array}$ & $\begin{array}{c}\text { Place of } \\
\text { arterial } \\
\text { catheter }\end{array}$ \\
\hline 1 & 1.7 & $4 \mathrm{~d}$ & 1 & RDS & UA \\
2 & 2.6 & $5 \mathrm{~d}$ & 1 & RDS & UA \\
3 & 2.8 & $3 \mathrm{~d}$ & 1 & Birth asphyxia & UA \\
4 & 3.6 & $1 \mathrm{~m}$ & 2 & Pneumonia & RA \\
5 & 3.8 & $4 \mathrm{~d}$ & 1 & MAS & UA \\
6 & 4.1 & $5 \mathrm{~d}$ & 1 & Birth asphyxia & UA \\
7 & 4.1 & $3.5 \mathrm{mo}$ & 2 & Postoperative & RA \\
8 & 4.7 & $5 \mathrm{mo}$ & 2 & Postoperative & RA \\
9 & 6.1 & $7 \mathrm{mo}$ & 3 & Postoperative & RA \\
10 & 10.6 & $1.4 \mathrm{y}$ & 4 & Pneumonia & FA \\
11 & 12.0 & $3 \mathrm{y}$ & 3 & Postoperative & RA \\
12 & 13.0 & $1.5 \mathrm{y}$ & 4 & Pneumonia & FA \\
13 & 15.0 & $2.6 \mathrm{y}$ & 4 & Postoperative & RA \\
14 & 15.7 & $2.8 \mathrm{y}$ & 4 & Postoperative & RA \\
15 & 16.0 & $4.0 \mathrm{y}$ & 4 & Infection & RA \\
\hline
\end{tabular}

RDS, respiratory distress syndrome; MAS, meconium aspiration syndrome; UA, umbilical artery; RA, radial artery; FA, femoral artery.

Subjects ordered by weight (kg) and age in days (d), months (mo), and years (y). Cuff sizes 1, 2, 3, and 4 fit fingers with a circumference of 22-26, $27-31,32-38$, and $39-46 \mathrm{~mm}$, respectively.

The local Ethics Committees approved the study. Informed consent was obtained from the parents of each child.

Instrumentation. The prototype for noninvasive finger arterial BP device for small children was based on Finapres technology (TNO Institute of Applied Physics, Biomedical Instrumentation, Amsterdam, The Netherlands). The prototype device consists of a finger BP cuff with a built-in infraredsensitive photocell, a pressure manometer, and an automatic unit for cuff inflation, and a monitor with digital display of systolic BP, diastolic BP, and heart rate. In the volume clamp method, the diameter of an artery under a cuff wrapped around the finger is kept constant (clamped) at a certain diameter during the pulsatile changes in arterial pressure. Minimal changes in diameter are detected by means of an infrared photoplethysmograph built into a finger cuff. If an increase in arterial diameter (systole) is detected, the finger cuff pressure is immediately increased by a rapid pressure servocontroller system to prevent the diameter change. At zero transmural pressure, the artery is "unloaded," which corresponds with the unstressed diameter of the vessel wall. As a result, finger cuff pressure equals intra-arterial pressure. However, the unloaded diameter is usually not constant during a measurement and has to be verified at intervals. Therefore, a software algorithm (Physiocal) is used to define and maintain the diameter at which the finger is clamped $(1,11)$.

We designed a set of four finger cuffs (Fig. 1) based on assessment of finger circumference and conicity of newborns, infants, and preschool children. Cuff sizes 1, 2, 3, and 4 fitted fingers with a circumference of 22-26, $27-31,32-38$, and $39-46 \mathrm{~mm}$, respectively. Measurements were performed with a prototype device based on Finapres technology in which adjustments to software parameters were made to accommodate the lower BP values and higher heart rates in young children. Author OS performed all measurements with the prototype device.

Data collection and analysis. Arterial BP was measured invasively through a 3-F umbilical catheter placed earlier in the lower aortic position (neonates) or through a peripheral 27-gauge infusion catheter in a peripheral artery. Arterial lines were placed for routine monitoring of vital functions and intensive care management. A $0.5 \mathrm{~mL} / \mathrm{h}$ infusion of heparinized physiologic saline solution was continuously flushed through the catheter. The arterial pressure wave form was measured with a HP M1006A pressure transducer (Hewlett Packard, Palo Alto, CA). Then it was subsequently digitized at a sample frequency of $200 \mathrm{~Hz}$ using an A/D converter and directly stored on the hard disk of the prototype device.

The finger cuff was applied on the middle finger. If the invasive catheter was placed in the radial artery, the finger cuff was applied at the contralateral side. The size of the finger cuff was determined by measuring the finger circumference. The cuffs were carefully wrapped round the middle phalanx. The finger arterial BP measurements were performed during 5-15 min. After stabilization of the noninvasive finger BP measurement (about $2 \mathrm{~min}$ ), we compared systolic, diastolic, and mean BP values. Finally, a pressure ramp was performed to evaluate the volume clamp methodology (Fig. 2). The BP

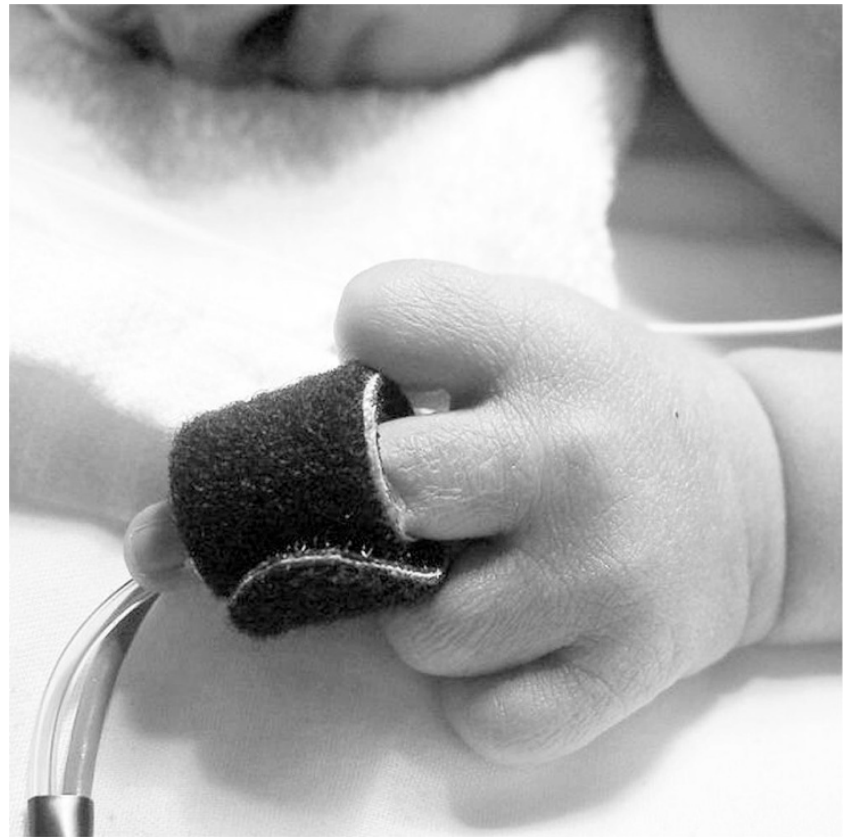

Figure 1. Illustration of a baby finger cuff.

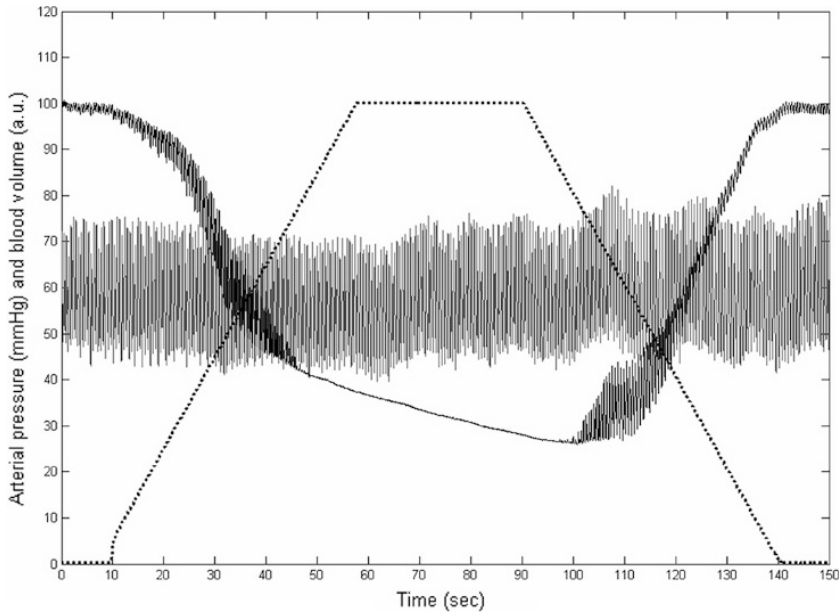

Figure 2. Illustration of a pressure ramp in a newborn. This graph illustrates the volume clamping technique. The $x$ axis represents time (s). The $y$ axis shows arterial blood pressure $(\mathrm{mm} \mathrm{Hg}$ ) as well as blood volume (arbitrary units). The gray line represents the invasive blood pressure, measured through a catheter in the umbilical artery. The black line represents the plethysmograph and can be interpreted as an approximation of blood volume under the cuff. The dashed line represents stepwise change of external applied pressure on the finger. As the external applied cuff pressure is increased, the plethysmograph shows maximal amplitude around the mean of the arterial blood pressure $(T=35 \mathrm{~s})$. When cuff pressure exceeds arterial systolic values, the pulsations in the plethysmograph disappear. As the cuff pressure is subsequently decreased, maximal pulsations of the plethysmograph reappear when cuff pressure is around the mean arterial blood pressure $(T=110 \mathrm{~s})$.

measurements were corrected for the hydrostatic height difference between the finger cuff and the arterial pressure sensor of the invasive line.

Statistics. The parametric data are expressed as mean $\pm \mathrm{SD}$, whereas nonparametric data are expressed as median and range. BP gain and offset were calculated by linear regression analysis between noninvasive and arterial values of systolic $\mathrm{BP}$ and diastolic $\mathrm{BP}$ : (beat-to-beat) $\mathrm{BP}_{\text {noninvasive }}=$ gain $\times$ (beat-to-beat) $\mathrm{BP}_{\text {invasive }}+$ offset (12). Bias in absolute and beat-to-beat $\mathrm{BP}$ values were calculated as the mean of difference between noninvasive and intra-arterial BP values and expressed as a function of mean values obtained from both methods, with its $95 \%$ limits of agreement. The root-mean-square 
error (RMSE) was calculated to quantify the difference between invasive and noninvasive waveforms. Statistical significance was accepted with a $p$ value less than 0.05 .

\section{RESULTS}

In most children, we obtained an arterial wave form at the first measurement attempt. In subject 5, it took several attempts to obtain a finger arterial wave form. Representative examples of the individual finger and invasive arterial BP waveforms are displayed in Figure 3. The mean RMSE value, as a quantitative measure for wave form analysis, was $3 \pm 2$ $\mathrm{mm} \mathrm{Hg}$.

During measurements, we observed no discomfort for the subjects. In most of the subjects, the fingertip colored temporarily dark red during the insufflations of the finger cuff. The normal color returned within a few seconds after the desufflation of the cuff. The coloring never gave reason to interrupt the measurement. None of the subjects sustained vascular damage or discomfort from the use of the finger cuff during the procedure.

A total of 2745 paired beats were analyzed, yielding a median of 134 analyzed paired beats per subject (range, 53-707). The mean invasive arterial BP was $82 \pm 18$ systolic and $47 \pm 12 \mathrm{~mm} \mathrm{Hg}$ diastolic, with a heart rate of $129 \pm 21$ beats per minute. The mean finger BP value was $77 \pm 22$ systolic and $45 \pm 16 \mathrm{~mm} \mathrm{Hg}$ diastolic. The correlation between noninvasive finger BP and the invasive arterial mean pressure showed a correlation coefficient of $0.79 \pm 0.19$ systolic and $0.74 \pm 0.24$ diastolic, with a correlation coefficient of $0.78 \pm 0.23$ for mean BP. The individual values of systolic, diastolic, and mean BP are presented in Table 2. The mean gain of absolute BP measurements was $0.9 \pm 0.2 \mathrm{~mm}$ $\mathrm{Hg} / \mathrm{mm} \mathrm{Hg}$ systolic and $0.8 \pm 0.2 \mathrm{~mm} \mathrm{Hg} / \mathrm{mm} \mathrm{Hg}$ diastolic, with offsets of +9 and $+8 \mathrm{~mm} \mathrm{Hg}$, respectively. The differences in BP values between the two methods as function of mean BP values of both methods are shown in Figure 4 for systolic BP (top) as well as mean (middle) and diastolic (bottom) BP values.
Table 2. Noninvasive finger cuff-derived $B P$ and invasive-derived $B P$ measurements

\begin{tabular}{ccc}
\hline Subject no. & Finger cuff BP & Invasive catheter BP \\
\hline 1 & $49 / 23(34)$ & $64 / 31(45)$ \\
2 & $51 / 30(40)$ & $59 / 38(48)$ \\
3 & $49 / 30(39)$ & $53 / 30(40)$ \\
4 & $72 / 45(58)$ & $84 / 43(62)$ \\
5 & $73 / 51(60)$ & $53 / 35(42)$ \\
6 & $66 / 45(56)$ & $71 / 45(57)$ \\
7 & $80 / 51(63)$ & $93 / 49(66)$ \\
8 & $55 / 25(40)$ & $90 / 41(64)$ \\
9 & $79 / 37(55)$ & $81 / 45(62)$ \\
10 & $85 / 58(70)$ & $100 / 57(75)$ \\
11 & $94 / 57(71)$ & $93 / 61(76)$ \\
12 & $65 / 25(38)$ & $94 / 45(62)$ \\
13 & $109 / 76(90)$ & $111 / 78(93)$ \\
14 & $105 / 57(77)$ & $98 / 53(74)$ \\
15 & $107 / 45(65)$ & $91 / 48(67)$
\end{tabular}

Mean values of systolic, diastolic, and mean (in parentheses) blood pressure (BP) of all subjects.

In Table 3, the beat-to-beat changes of Finapres and arterial BP signals are presented. Note that almost no bias was observed between both methods for the beat-to-beat BP values. Details of the individual $95 \%$ limits of agreement are shown in Table 3. The mean correlation coefficients were $0.82 \pm 0.18$ systolic and $0.75 \pm 0.21$ diastolic, respectively. The mean gain of beat-to-beat BP measurements was $0.8 \pm 0.3 \mathrm{~mm}$ $\mathrm{Hg} / \mathrm{mm} \mathrm{Hg}$ systolic and $0.7 \pm 0.2$ diastolic, with offsets of 0 $\mathrm{mm} \mathrm{Hg}$.

Analyzing the pressure ramps showed that three measurements were related to errors in the measurement procedure. We address these aspects in the Discussion section. The measurement in subject 5 (loose cuff application, measurement indicated as a square in Fig. 3) showed a difference of $+18 \mathrm{~mm} \mathrm{Hg}$, i.e., the noninvasively determined mean BP in the finger was $18 \mathrm{~mm} \mathrm{Hg}$ higher than the invasively measured BP. The measurements in subjects 8 and 12 (wrong set-point, measurement indicated as triangles in Fig. 3) showed a mean

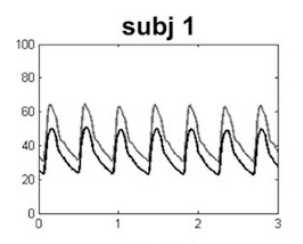

subj 6

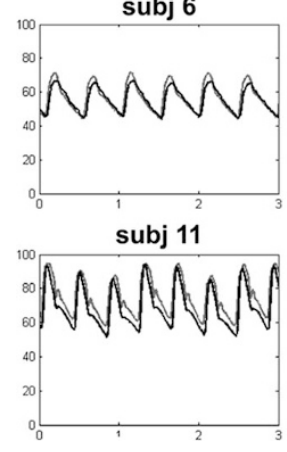

subj 2

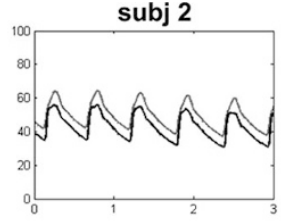

subj 7

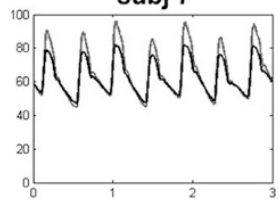

subj 12

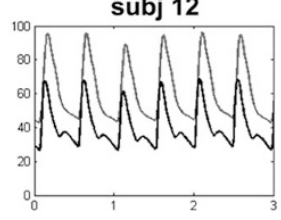

subj 3

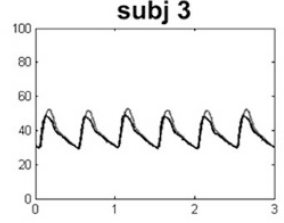

subj 8

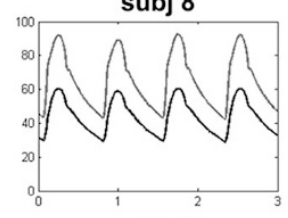

subj 13

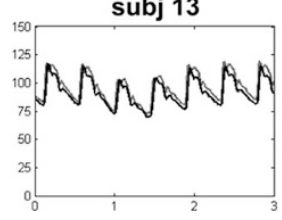

subj 4

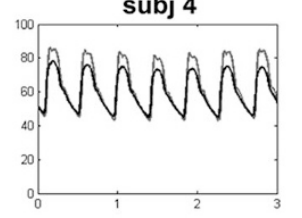

subj 9

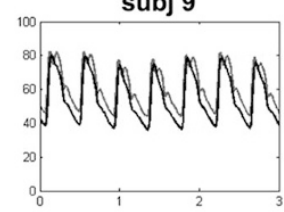

subj 14

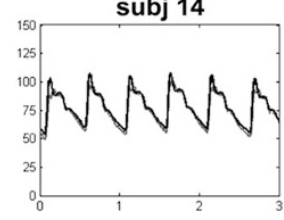

subj 5

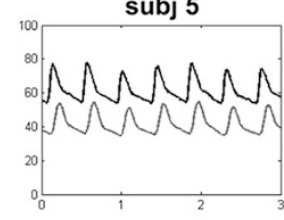

subj 10

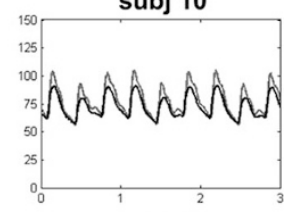

subj 15

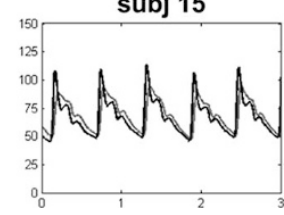

Figure 3. Waveforms of finger cuff-derived arterial BP and invasive arterial BP. The finger cuff-derived arterial pressure wave form is shown in black. The invasively obtained arterial pressure is shown in gray. $x$ axis, time in seconds; $y$ axis, pressure in $\mathrm{mm} \mathrm{Hg}$. Note that for subjects $10,13,14$, and 15 the $y$ scale is from 0 to 150; for all others, the $y$ scale is from 0 to $100 \mathrm{~mm} \mathrm{Hg}$. 

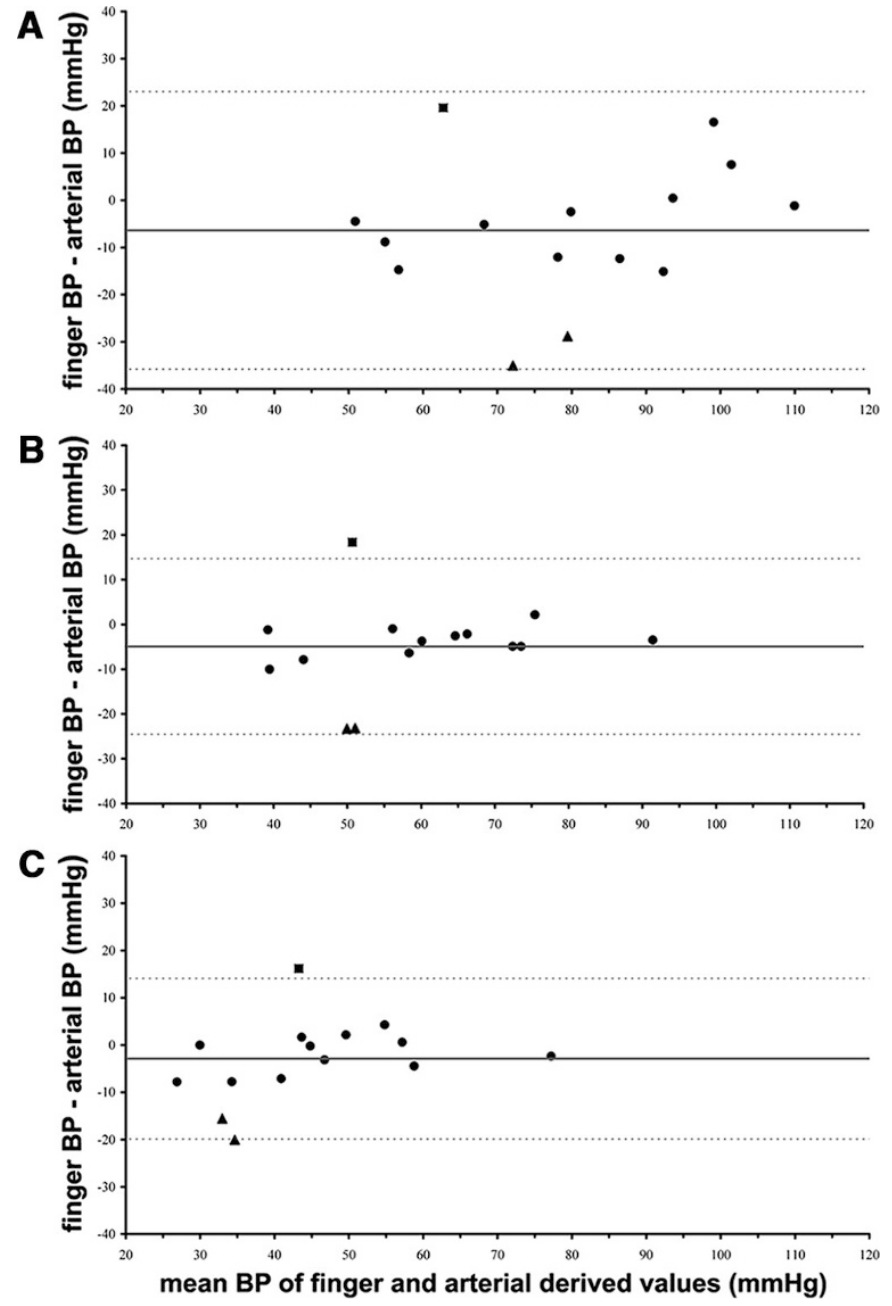

Figure 4. Bias in absolute BP values. Bland-Altman plots displaying mean differences for individual subjects $(\bullet)$ between finger BP and invasive BP measurements for systolic $(A)$, mean $(B)$, and diastolic $(C)$ BP values $(\mathrm{mm}$ $\mathrm{Hg}$ ) versus average of the two pressures $(\mathrm{mm} \mathrm{Hg})$. The solid line represents the mean difference for all subjects between both methods of measurements, and the dashed lines represent $95 \%$ limits of agreement. Three measurements were related to errors in the measurement procedure [loose cuff application $(\square)]$; set-point error of algorithm $(\mathbf{\Delta})$.

Table 3. Beat-to-beat changes

\begin{tabular}{crrrrr}
\hline \multirow{2}{*}{$\begin{array}{c}\text { Subject } \\
\text { no. }\end{array}$} & \multicolumn{2}{c}{ Bias } & & \multicolumn{2}{c}{$95 \%$ limits of agreement } \\
\cline { 2 - 3 } \cline { 5 - 6 } & SBP & DBP & & SBP & DBP \\
\hline 1 & 0.0 & 0.0 & & -1.8 to +1.8 & -1.5 to +1.5 \\
2 & -0.1 & -0.1 & & -1.8 to +1.6 & -1.4 to +1.2 \\
3 & 0.0 & 0.0 & & -1.5 to +1.5 & -1.2 to +1.2 \\
4 & -0.3 & -0.1 & & -2.1 to +1.5 & -1.3 to +1.1 \\
5 & -0.1 & 0.0 & & -2.6 to +2.4 & -1.7 to +1.7 \\
6 & 0.0 & 0.0 & & -2.3 to +2.3 & -1.7 to +1.7 \\
7 & -0.1 & 0.0 & & -5.4 to +5.2 & -2.5 to +2.5 \\
8 & -0.2 & -0.1 & & -5.3 to +4.9 & -3.6 to +3.4 \\
9 & 0.0 & 0.0 & & -3.3 to +3.3 & -2.0 to +2.0 \\
10 & 0.0 & -0.1 & & -4.1 to +4.1 & -4.9 to +4.7 \\
11 & 0.0 & 0.0 & & -3.2 to +3.2 & -1.6 to +1.6 \\
12 & 0.0 & 0.0 & & -2.4 to +2.4 & -1.9 to +1.9 \\
13 & 0.0 & 0.0 & & -2.3 to +2.3 & -1.7 to +1.7 \\
14 & -0.1 & 0.0 & -4.2 to +4.0 & -4.8 to +4.8 \\
15 & -0.1 & 0.0 & & -8.3 to +8.1 & -3.1 to +3.1 \\
\hline
\end{tabular}

Bias (mean of the difference) and 95\% limits of agreement for systolic (SBP) and diastolic (DBP) beat-to-beat changes, respectively. Data expressed in $\mathrm{mm} \mathrm{Hg}$.
BP difference of $-24 \mathrm{~mm} \mathrm{Hg}$ between the two methods, i.e., the noninvasively determined mean BP in the finger was 24 $\mathrm{mm} \mathrm{Hg}$ lower than the invasively measured BP. Excluding these erroneous measurements resulted in clinically acceptable measurement bias $(-3.8 \mathrm{~mm} \mathrm{Hg})$ and $95 \%$ limits of agreement $(-10.4$ to $+2.8 \mathrm{~mm} \mathrm{Hg})$, and the correlation increased to $0.84 \pm 0.18$ for mean $\mathrm{BP}$ values.

\section{DISCUSSION}

The Finapres technology is already established over more than $25 \mathrm{y}$ for continuous, noninvasive measurement of arterial BP using a compact and simple cuff on the finger (13). However, the Finapres technology is validated only in adults and children $>6$ y $(4,12)$. This is the first study that suggests that continuous finger BP waveforms can be obtained by a noninvasive technique using appropriate small finger cuffs in very young children, aged $0-4 \mathrm{y}$.

Previously, several publications have shown the feasibility of BP measurements with the Finapres method in newborns by applying the (commercially available) smallest adult size finger cuff around the wrist (6-9). However, finger cuff application has an important advantage over wrist application, mainly related to the superficial location of the digital arteries and circular shape of the finger. The radial artery is deeply hidden underneath the skin, and therefore, photoplethysmography will have difficulties to catch the arterial pulsation between the s.c. and bony structures. In contrast, the palmar and dorsal digital arteries of the finger are located superficially. Small anastomoses occur mainly in the s.c. tissue of the fingertip and near the interphalangeal joints. Between the interphalangeal joints, precisely at cuff application, the superficially located arteries have straight axes without important collaterals. In addition, volume clamping technique is more easily applied in the case of an object with a circular shape, e.g., the finger.

The application of noninvasive continuous BP measurement in newborn and infants are several. In contrast to traditionally noninvasive oscillatory BP methods, continuous finger BP measurement provides the ability to measure continuous cardiac output from pulse contour analysis of the finger arterial wave form (14). Continuous monitoring of BP is essential in the evaluation of patients with cardiovascular abnormalities and autonomic dysfunction (15). Newborns with apparent life-threatening events and preschool children with a postural orthostatic hypotension or obstructive sleep apnea syndrome show reduced baroreceptor-mediated heart rate response (16-18). Low birth weight is inversely associated with systolic BP in children and adults (19). Adolescents who had lower birth weight showed higher 24-h BP values as well as higher BP variability, indicating abnormal cardiovascular control (20). Without the need of invasive arterial catheters, continuous noninvasive finger BP method will give insight in (short-term) BP regulation.

Several methodological considerations have to be addressed. First, the invasive arterial pressures were measured at different sites of the arterial tree. Because of reflections of the pressure wave at different point in the arterial tree and resistance to flow, the BP wave form in the finger artery may differ 
in shape and mean value from the BP in the aorta and other measurement sites (21). However, we observed (Fig. 3) in most cases no clear difference in the peripheral (finger) BP wave form compared with the more central (arterial) BP wave form. Indeed, we found low RMSE values in newborns, indicating that both waveforms are very similar. This observation corresponds with a study performed in critically ill newborns (22). In this study, arterial BP waveforms were documented at two sites along the arterial tree of the neonate: in the radial and posterior tibial arteries. From this study, it is concluded that radial and posterior tibial artery waveforms in newborns seem to have a similar appearance as the waveforms obtained in the large central arteries. An explanation of this phenomenon might be the close proximity of the radial and posterior tibial artery to the central aorta and femoral artery respectively, because of the small and short limbs of the neonate. In addition, we observed a good correlation between finger cuff-derived BP values and arterial BP values. Apparently, compared with adults, the peripheral BP waveforms and values in infants seem to deviate less from central pressure (22,23).

Second, we found a good correlation coefficient and almost no bias between beat-to-beat values between both methods. This indicates that changes in BP values are well tracked by the noninvasive BP method. However, the gain below $1 \mathrm{~mm}$ $\mathrm{Hg} / \mathrm{mm} \mathrm{Hg}$ indicates increasing negative bias with increasing beat-to-beat changes. The algorithm has to be improved to correct for this error.

Third, in very young children, the cuff application and Physiocal algorithm seems to be critical. Therefore, several small finger cuffs were designed (circumference of 22-26, 27-31, 32-38, and 39-46 mm, respectively) for the different pediatric age groups. If a finger cuff is loosely applied to the finger, BP will be higher because of the limiting compliance of the bladder within the cuff. The Physiocal algorithm includes a protocol for the automated determination and periodic adjustment of the arterial unloaded diameter. It explores the pressure-diameter relation by analyzing the plethysmogram at a number of steady pressure levels at regular intervals (1). The methodology of volume clamping is illustrated in Figure 2. Review of the Physiocal procedure in subject 5 showed that increase of the cuff pressure above systolic BP still resulted in pulsations of the plethysmogram, indicating loose cuff application. Indeed, the finger cuff application in subject 5 was only successful after several attempts of applications. Review of the Physiocal procedure in subjects 8 and 12 showed evidence of a wrong set-point of the algorithm. Normally, the unloaded diameter is close to the average diameter at a pressure where the amplitude of the pulsations in the plethysmogram is largest (Fig. 2). However, in subjects 8 and 12, during stepwise increase of the cuff pressure, the plethysmographic pulsations were not maximal around the mean BP, thus indicating a wrong set-point. The arterial unloaded diameter (thus setpoint) may be influenced by a different elasticity of the artery, smooth muscle cell function, or hematocrit. We conclude that the current adult set-point algorithm may be improved for measurements in very young children. Excluding these three erroneous measurements resulted in clinically acceptable measurement bias $(-3.8 \mathrm{~mm} \mathrm{Hg})$ and $95 \%$ limits of agreement $(-10.4$ to $+2.8 \mathrm{~mm} \mathrm{Hg})$.

Fourth, artifacts due to movement of the fingers were generally a problem of the first minutes of the measurement. Within minutes, the newborn or infant was accommodated to the oscillating finger cuff and relaxed their fingers. Therefore, measurements were performed after a stabilization period of several minutes. In addition, cold fingers and other vasospastic conditions have to be prevented, because these conditions will affect the peripheral circulation and thus the finger BP measurement.

In conclusion, the method of volume clamping on the finger is feasible in very young children. In these children, the pressure curves in the finger show good resemblances with the central arterial waveforms. Changes in BP are well tracked by the noninvasive method. Cuff design and cuff application are critical. The set-point algorithm needs to be optimized for the application in very young children. To make the system ambulatory, appropriate for long term, continuous monitoring a trade-off has to be made between the signal amplitude and the applied external pressure of the finger cuff.

Acknowledgments. We thank S. Houterman, consultant in biostatistics, Máxima Medical Center, Veldhoven, The Netherlands, for her critical review of the article. BMEYE (Cardiovascular Monitoring, Academic Medical Center, Amsterdam, The Netherlands) provided the finger cuffs and modifications of the software of the Finapres device for the study.

\section{REFERENCES}

1. Wesseling KH 1996 Finger arterial pressure measurement with Finapres. Z Kardiol $85: 38-44$

2. Parati G, Casadei R, Groppelli A, Di Rienzo M, Mancia G 1989 Comparison of finger and intra-arterial blood pressure monitoring at rest and during laboratory testing. Hypertension 13:647-655

3. Penaz J, Voigt A, Teichmann W 1976 [Contribution to the continuous indirect blood pressure measurement]. Z Gesamte Inn Med 31:1030-1033

4. Tanaka H, Thulesius O, Yamaguchi H, Mino M, Konishi K 1994 Continuous non-invasive finger blood pressure monitoring in children. Acta Paediatr 83:646652

5. de Jong-de Vos van Steenwijk CC, Wieling W, Harms MP, Wesseling KH 1997 Variability of near-fainting responses in healthy 6-16-year-old subjects. Clin Sci (Lond) 93:205-211

6. Andriessen P, Schoffelen RL, Berendsen RC, de Beer NA, Oei SG, Wijn PF, Blanco CE 2004 Noninvasive assessment of blood pressure variability in preterm infants. Pediatr Res 55:220-223

7. Drouin E, Gournay V, Calamel J, Mouzard A, Roze JC 1997 Feasibility of using finger arterial pressure in neonates. Arch Dis Child Fetal Neonatal Ed 77:F139F140

8. Drouin E, Gournay V, Calamel J, Mouzard A, Roze JC 1997 Assessment of spontaneous baroreflex sensitivity in neonates. Arch Dis Child Fetal Neonatal Ed 76:F108-F112

9. Yiallourou SR, Walker AM, Horne RS 2006 Validation of a new noninvasive method to measure blood pressure and assess baroreflex sensitivity in preterm infants during sleep. Sleep 29:1083-1088

10. Parati G, Di Rienzo M, Bertinieri G, Pomidossi G, Casadei R, Groppelli A, Pedotti A, Zanchetti A, Mancia G 1988 Evaluation of the baroreceptor-heart rate reflex by 24-hour intra-arterial blood pressure monitoring in humans. Hypertension 12:214222

11. Bogert LW, van Lieshout JJ 2005 Non-invasive pulsatile arterial pressure and stroke volume changes from the human finger. Exp Physiol 90:437-446

12. Triedman JK, Saul JP 1994 Comparison of intraarterial with continuous noninvasive blood pressure measurement in postoperative pediatric patients. J Clin Monit 10:11-20

13. Imholz BP, Wieling W, van Montfrans GA, Wesseling KH 1998 Fifteen years experience with finger arterial pressure monitoring: assessment of the technology. Cardiovasc Res 38:605-616 
14. Jellema WT, Imholz BP, van Goudoever J, Wesseling KH, van Lieshout JJ 1996 Finger arterial versus intrabrachial pressure and continuous cardiac output during head-up tilt testing in healthy subjects. Clin Sci (Lond) 91:193-200

15. Harrington C, Kirjavainen T, Teng A, Sullivan CE 2001 Cardiovascular responses to three simple, provocative tests of autonomic activity in sleeping infants. J Appl Physiol 91:561-568

16. Axelrod FB, Chelimsky GG, Weese-Mayer DE 2006 Pediatric autonomic disorders. Pediatrics 118:309-321

17. Harrington C, Kirjavainen T, Teng A, Sullivan CE 2002 Altered autonomic function and reduced arousability in apparent life-threatening event infants with obstructive sleep apnea. Am J Respir Crit Care Med 165:1048-1054

18. Amin RS, Carroll JL, Jeffries JL, Grone C, Bean JA, Chini B, Bokulic R, Daniels SR 2004 Twenty-four-hour ambulatory blood pressure in children with sleep-disordered breathing. Am J Respir Crit Care Med 169:950-956
19. Law CM, Shiell AW 1996 Is blood pressure inversely related to birth weight? The strength of evidence from a systematic review of the literature. J Hypertens 14:935-941

20. Lurbe E, Torro I, Rodriguez C, Alvarez V, Redon J 2001 Birth weight influences blood pressure values and variability in children and adolescents. Hypertension 38:389-393

21. Kleinman B 1989 Understanding natural frequency and damping and how they relate to the measurement of blood pressure. J Clin Monit 5:137-147

22. Gevers M, Hack WW, Ree EF, Lafeber HN, Westerhof N 1993 Arterial blood pressure wave forms in radial and posterior tibial arteries in critically ill newborn infants. J Dev Physiol 19:179-185

23. Gevers M, Van Genderingen HR, Lafeber HN, Hack WW 1995 Radial artery blood pressure measurement in neonates: an accurate and convenient technique in clinical practice. J Perinat Med 23:467-475 